Проблеми математичного моделювання та теорії диференціальних рівнянь

УДК 517.977.56

\title{
НЕОБХІДНІ УМОВИ ОПТИМАЛЬНОСТІ ДЛЯ ЗАДАЧІ КЕРУВАННЯ ПАРАБОЛІЧНОЮ СИСТЕМОЮ З НЕОБМЕЖЕНИМИ КОЕФІЩІЕНТАМИ
}

\author{
С. О. Горбонос*, П. І. Когут**
}

*Дніпропетровсъкий національний університет, кафедра диференціальних рівнянь, вул. Козакова, 18/14, ДНУ, 49010 Дніпропетровсък, E-таil: gorbonos.so@gmail.com

** Дніпропетровсъкий національний університет, кафедра диференціальних рівнянь, вул. Козакова, 18/14, ДНУ, 49010 Дніпропетровсък, E-mail: p.kogut@i.uа

Встановлено необхідні умови оптимальності для задачі оптимального керування параболічною системою з необмеженими коефіцієнтами із залученням концепції узагальненої правої похідної за напрямом та поняття квазіспряженого стану.

Ключові слова: задача оптимального керування, функціонал Лагранжа, узагальнена права похідна за напрямом, квазіспряжений оператор, необхідні умови оптимальності.

\section{1. Вступ}

Розглянуто задачу оптимального керування параболічною системою з необмеженими коефіцієнтами. Характерна особливість даного класу задач полягає в тому, що матриця потоку є кососиметрична, а її коефіцієнти належать до простору $L^{2}$. За таких умов не можна отримати необхідні умови оптимальності, застосувавши класичні результати Йоффе і Тихомирова [1]. Тому метою даної роботи було встановити необхідні умови оптимальності для поставленої задачі керування, залучаючи концепцію узагальненої правої похідної за напрямом та поняття квазіспряженого стану [3].

\section{2. Постановка задачі та попердні означення}

Об'єктом дослідження виступає така задача оптимального керування:

$$
\begin{gathered}
y_{t}-\operatorname{div}(\nabla y+A(x) \nabla y)=f \quad \text { на } \Omega \times[0, T] \\
y=0 \quad \text { на } \quad \Gamma_{1} \times[0, T], \quad \partial y / \partial \nu_{A}=u \quad \text { на } \quad \Gamma_{2} \times[0, T], \\
y\left(t_{0}, x\right)=y_{0} \in L^{2}(\Omega) \quad \text { на } \Omega, \\
I(u, y)=\left\|y-y_{d}\right\|_{L^{2}\left(0, T ; H_{0}^{1}(\Omega)\right)}^{2}+\|u\|_{L^{2}\left(0, T ; L^{2}\left(\Gamma_{2}\right)\right)}^{2} \rightarrow \inf ,
\end{gathered}
$$

(C) С. О. Горбонос, П. I. Когут, 2014 
де $f \in L^{2}\left(0, T ; H^{-1}(\Omega)\right) ; u \in L^{2}\left(0, T ; L^{2}\left(\Gamma_{2}\right)\right) ; A(x) \in L^{2}\left(\Omega ; \mathbb{S}^{N}\right)$ - кососиметрична матриця, тобто $a_{i j}=-a_{j i}$ i $a_{i i}=0$.

Нехай $1 \leqslant p<\infty$, далі через $L^{p}([0, T], V)$ будемо позначати множину всіх вимірних функцій $u:[0, T] \rightarrow V$ таких, що

$$
\|u\|_{L^{p}([0, T], V)}=\left(\int_{0}^{T}\|u(t)\|_{V}^{p} d t\right)^{1 / p}<\infty
$$

тут інтеграл наведено в сенсі Бохнера [4]. У випадку, коли $p=\infty$, маємо

$$
\|u\|_{L^{\infty}([0, T], V)}=\operatorname{ess} \sup _{0 \leqslant t \leqslant T}\|u(t)\|_{V}<\infty .
$$

Оскільки $A(x) \in L^{2}\left(\Omega ; \mathbb{S}^{N}\right)$, то матриця $I+A(x)$ породжена необмеженою білінійною формою на $L^{2}\left(\Omega ; \mathbb{R}^{N}\right)$, з огляду на це введемо до розгляду множину.

Означення 2.1. Будемо казати, що елемент $y \in L^{2}\left(0, T ; H_{0}^{1}(\Omega)\right)$ належить до множини $D$, якщо

$$
\left|\int_{0}^{T} \int_{\Omega}(\nabla \varphi, A \nabla y)_{\mathbb{R}^{N}} d x d t\right| \leqslant c(y)\left(\int_{0}^{T} \int_{\Omega}|\nabla \varphi|^{2} d x d t\right)^{1 / 2}
$$

для будь-якого $\varphi \in C^{\infty}\left(0, T ; C_{0}^{\infty}(\Omega)\right)$, де $c(y)$ - стала, яка залежить від $y$.

Далі введемо до розгляду форму

$$
[y, \varphi]=\int_{0}^{T} \int_{\Omega}(\nabla \varphi, A \nabla y)_{\mathbb{R}^{N}} d x d t \forall y \in D, \forall \varphi \in C^{\infty}\left(0, T ; C_{0}^{\infty}(\Omega)\right)
$$

і означимо білінійну форму $[y, \varphi]$ для всіх $\varphi \in L^{2}\left(0, T ; H_{0}^{1}(\Omega)\right)$ за правилом

$$
[y, \varphi]=\lim _{\varepsilon \rightarrow \infty}\left[y, \varphi_{\varepsilon}\right]
$$

де $\left\{\varphi_{\varepsilon}\right\}_{\varepsilon>0} \subset C^{\infty}\left(0, T ; C_{0}^{\infty}(\Omega)\right)$ і $\varphi_{\varepsilon} \rightarrow \varphi$ сильно в $L^{2}\left(0, T ; H_{0}^{1}(\Omega)\right)$.

Означення 2.2. [2] Допустимою парою задачі оптимального керування (2.1)-(2.4) будемо називати пару $(u, y)$, якщо $u \in L^{2}\left(0, T ; L^{2}\left(\Gamma_{2}\right)\right), y \in D$ i пара $(u, y)$ задовольняє енергетичну рівність

$$
\begin{gathered}
\int_{0}^{T} \int_{\Omega} y_{t} \varphi d x d t+\int_{0}^{T} \int_{\Omega}(\nabla \varphi, \nabla y+A \nabla y)_{\mathbb{R}^{N}} d x d t \\
=\int_{0}^{T} \int_{\Omega} f \varphi d x d t+\int_{0}^{T} \int_{\Gamma_{2}} u \varphi d \mathcal{H}^{N-1} d t
\end{gathered}
$$

для будь-якого $\varphi \in C^{\infty}\left(0, T ; C_{0}^{\infty}(\Omega)\right)$. Далі $\Xi-$ це множина всіх допустимих пар задачі (2.1)-(2.4). 


\section{3. Поняття узагальненої правої похідної за напрямом та їі властивості}

Розглянемо функціонал Лагранжа і введемо поняття узагальненої правої похідної за напрямом для задачі (2.1)-(2.4).

Нехай $F(A, y)=y_{t}-\operatorname{div}(\nabla y+A(x) \nabla y)$, тоді функціонал Лагранжа матиме вигляд

$$
\begin{aligned}
L(u, y, \lambda, \varphi)=\lambda I(u, y)+\langle F(A, y), \varphi\rangle_{L^{2}\left(0, T ; H^{-1}(\Omega)\right) ; L^{2}\left(0, T ; H_{0}^{1}(\Omega)\right)} & \\
& -\langle f, \varphi\rangle_{L^{2}\left(0, T ; H^{-1}(\Omega)\right) ; L^{2}\left(0, T ; H_{0}^{1}(\Omega)\right)},
\end{aligned}
$$

де $\lambda \in \mathbb{R}_{+}, \varphi \in C^{\infty}\left(0, T ; C_{0}^{\infty}(\Omega)\right)$ i

$$
\begin{gathered}
\langle F(A, y), \varphi\rangle_{L^{2}\left(0, T ; H^{-1}(\Omega)\right) ; L^{2}\left(0, T ; H_{0}^{1}(\Omega)\right)}=\int_{0}^{T} \int_{\Omega} y_{t} \varphi d x d t \\
+\int_{0}^{T} \int_{\Omega}(\nabla \varphi, \nabla y)_{\mathbb{R}^{N}} d x d t+\int_{0}^{T} \int_{\Omega}(\nabla \varphi, A \nabla y)_{\mathbb{R}^{N}} d x d t-\int_{0}^{T} \int_{\Gamma_{2}} u \varphi d \mathcal{H}^{N-1} d t .
\end{gathered}
$$

Беручи до уваги зауваження 3.1 [2], отримаємо, що функціонал (3.1) може бути продовжений для всіх $\varphi \in C^{\infty}\left(0, T ; C_{0}^{\infty}(\Omega)\right)$ за правилом (2.7). У результаті для кожного $u \in L^{2}\left(0, T ; L^{2}\left(\Gamma_{2}\right)\right)$ продовжений функціонал $\widehat{L}$ : $L^{2}\left(0, T ; L^{2}\left(\Gamma_{2}\right)\right) \times D \times \mathbb{R}_{+} \times L^{2}\left(0, T ; H_{0}^{1}(\Omega)\right)$ матиме такий вигляд:

$$
\begin{aligned}
\widehat{L}(u, y, \lambda, p)=\lambda I(u, y) & +\int_{0}^{T} \int_{\Omega} y_{t} p d x d t \\
& +\int_{0}^{T} \int_{\Omega}(\nabla p, \nabla y)_{\mathbb{R}^{N}} d x d t+[y, p] \\
& -\langle f, p\rangle_{L^{2}\left(0, T ; H^{-1}(\Omega)\right) ; L^{2}\left(0, T ; H_{0}^{1}(\Omega)\right)}-\int_{0}^{T} \int_{\Gamma_{2}} u \varphi d \mathcal{H}^{N-1} d t .
\end{aligned}
$$

Звідси, оскільки білінійна форма $\int_{0}^{T} \int_{\Omega}(\nabla p, A(x) \nabla y)_{\mathbb{R}^{N}} d x d t$ необмежена, то продовження функціоналу Лагранжа $\widehat{L}(u, y, \lambda, p)$ не є диференційовним за Гато. Крім того, не можна навіть стверджувати, що відображення $y \rightarrow \widehat{L}(u, y, \lambda, p)$ має праву похідну за напрямом $h$

$$
D_{y}^{+} \widehat{L}(u, y, \lambda, p, h)=\lim _{\theta \rightarrow+0} \frac{\widehat{L}(u, y+\theta h, \lambda, p)-\widehat{L}(u, y, \lambda, p)}{\theta} .
$$


Дійсно, для заданого $h \in L^{2}\left(0, T ; H_{0}^{1}(\Omega)\right)$ і $\theta \in[0,1]$ не можна стверджувати, що $y+\theta h \in D$, оскільки структура $D$ невідома, навіть якщо величина $\theta$ достатньо мала.

Враховуючи, що множина допустимих розв'язків поставленої задачі $\Xi$ складається з пар $(u, y) \in \Xi$ таких, що $A \in L^{2}\left(\Omega ; \mathbb{S}^{N}\right)$, то відображення $y \mapsto F(A, y)$ не $\epsilon$ неперервно диференційовне в околі $y$. Це говорить про те, що не можна застосувати добре відомі результати Йоффе і Тихомирова, щоб отримати необхідні умови оптимальності [1]. Отже, враховуючи вищесказане, наведемо таку концепцію.

Означення 3.1. [3] Будемо казати, що відображення $y \mapsto \widehat{L}(u, y, \lambda, p)$ має узагальнену праву похідну за напрямом $h \in L^{2}\left(0, T ; H_{0}^{1}(\Omega)\right)$ у точці $(u, y, \lambda, p) \in$ $L^{2}\left(0, T ; L^{2}\left(\Gamma_{2}\right)\right) \times D \times \mathbb{R}_{+} \times L^{2}\left(0, T ; H_{0}^{1}(\Omega)\right)$, якщо праву похідну за гладким напрямом $\varphi \in C^{\infty}\left(0, T ; C_{0}^{\infty}(\Omega)\right)$ можно неперервно продовжити для $\varphi=h \in$ $L^{2}\left(0, T ; H_{0}^{1}(\Omega)\right)$, тобто

$$
D_{y}^{+} \widehat{L}(u, y, \lambda, p, h)=\lim _{\varepsilon \rightarrow 0} D_{y}^{+} \widehat{L}\left(u, y, \lambda, p, \varphi_{\varepsilon}\right)
$$

для будь-яких $\left\{\varphi_{\varepsilon}\right\}_{\varepsilon>0} \subset C^{\infty}\left(0, T ; C_{0}^{\infty}(\Omega)\right)$ таких, що:

$$
\begin{gathered}
\varphi_{\varepsilon} \rightarrow h \quad \text { сильно в } \quad L^{2}\left(0, T ; H_{0}^{1}(\Omega)\right), \\
\left(\varphi_{\varepsilon}\right)_{t}^{\prime} \rightarrow(h)_{t}^{\prime} \quad \text { сильно в } \quad L^{2}\left(0, T ; H^{-1}(\Omega)\right) .
\end{gathered}
$$

Тепер застосуємо цю концепцію до функціоналу Лагранжа $\widehat{L}(u, y, \lambda, p)$.

Лема 3.1. Нехай задано четвірку $(u, y, \lambda, p) \in L^{2}\left(0, T ; L^{2}\left(\Gamma_{2}\right)\right) \times D \times \mathbb{R}_{+} \times$ $L^{2}\left(0, T ; H_{0}^{1}(\Omega)\right)$ таку, що $p \in D$, тоді для кожного напряму $h$ простору $L^{2}\left(0, T ; H_{0}^{1}(\Omega)\right)$ узагалвнена права похідна за напрямом $D_{y}^{+} \widehat{L}(u, y, \lambda, p, h)$ icнуе і мае вигляд

$$
\begin{aligned}
D_{y}^{+} \widehat{L}(u, y, \lambda, p, h)=2 \lambda \int_{0}^{T} \int_{\Omega}\left(y-y_{d}\right) h d x d t & +\int_{0}^{T} \int_{\Omega} h_{t}^{\prime} p d x d t \\
& +\int_{0}^{T} \int_{\Omega}(\nabla p, \nabla h)_{\mathbb{R}^{N}} d x d t-[p, h] .
\end{aligned}
$$

Доведення. Нехай для заданої четвірки $(u, y, \lambda, p)$ і для $\varepsilon>0$ послідовність функцій $\left\{\varphi_{\varepsilon}\right\} \subset C^{\infty}\left(0, T ; C_{0}^{\infty}(\Omega)\right) \in$ така, що

$$
\begin{gathered}
\varphi_{\varepsilon} \rightarrow h \quad \text { сильно в } \quad L^{2}\left(0, T ; H_{0}^{1}(\Omega)\right), \\
\left(\varphi_{\varepsilon}\right)_{t}^{\prime} \rightarrow(h)_{t}^{\prime} \quad \text { сильно в } \quad L^{2}\left(0, T ; H^{-1}(\Omega)\right) .
\end{gathered}
$$


Тоді

$$
\begin{gathered}
D_{y}^{+} \widehat{L}\left(u, y, \lambda, p, \varphi_{\varepsilon}\right)=\lim _{\theta \rightarrow+0} \frac{\widehat{L}\left(u, y+\theta \varphi_{\varepsilon}, \lambda, p\right)-\widehat{L}(u, y, \lambda, p)}{\theta} \\
=\lim _{\theta \rightarrow+0} \frac{1}{\theta}\left\{\lambda\left\|y+\theta \varphi_{\varepsilon}-y_{d}\right\|_{L^{2}\left(0, T ; H_{0}^{1}(\Omega)\right)}^{2}+\lambda\|u\|_{L^{2}\left(0, T ; L^{2}\left(\Gamma_{2}\right)\right)}^{2}\right. \\
+\int_{0}^{T} \int_{\Omega}\left(y+\theta \varphi_{\varepsilon}\right)_{t}^{\prime} p d x d t+\int_{0}^{T} \int_{\Omega}\left(\nabla p, \nabla\left(y+\theta \varphi_{\varepsilon}\right)\right)_{\mathbb{R}^{N}} d x d t \\
+\left[y+\theta \varphi_{\varepsilon}, \psi_{\delta}\right]-\langle f, p\rangle_{L^{2}\left(0, T ; H^{-1}(\Omega)\right), L^{2}\left(0, T ; H_{0}^{1}(\Omega)\right)}-\int_{0}^{T} \int_{\Gamma_{2}} u p d \mathcal{H}^{N-1} d t \\
-\lambda\left\|y-y_{d}\right\|_{L^{2}\left(0, T ; H_{0}^{1}(\Omega)\right)}^{2}-\lambda\|u\|_{L^{2}\left(0, T ; L^{2}\left(\Gamma_{2}\right)\right)}^{2}-\int_{0}^{T} \int_{\Omega} y_{t} p d x d t \\
\left.-[y, p]+\langle f, p\rangle_{L^{2}\left(0, T ; H^{-1}(\Omega)\right), L^{2}\left(0, T ; H_{0}^{1}(\Omega)\right)}+\int_{0}^{T} \int_{\Gamma_{2}} u p d \mathcal{H}^{N-1} d t\right\} \\
=2 \lambda \int_{0}^{T} \int_{\Omega}\left(y-y_{d}\right) \varphi_{\varepsilon} d x d t+\int_{0}^{T} \int_{\Omega}\left(\varphi_{\varepsilon}\right)_{t}^{\prime} p d x d t \\
+\int_{0}^{T} \int_{\Omega}\left(\nabla p, \nabla \varphi_{\varepsilon}\right)_{\mathbb{R}^{N}} d x d t+J
\end{gathered}
$$

де

$$
\begin{gathered}
J=\lim _{\theta \rightarrow+0} \frac{\lim _{\delta \rightarrow 0}\left[y+\theta \varphi_{\varepsilon}, \psi_{\delta}\right]-[y, p]}{\theta} \\
{\left[y+\theta \varphi_{\varepsilon}, \psi_{\delta}\right]=\int_{0}^{T} \int_{\Omega}\left(\nabla \psi_{\delta}, A(x) \nabla\left(y+\theta \varphi_{\varepsilon}\right)\right)_{\mathbb{R}^{N}} d x d t,} \\
{[y, p]=\int_{0}^{T} \int_{\Omega}(\nabla p, A(x) \nabla y)_{\mathbb{R}^{N}} d x d t}
\end{gathered}
$$

для будь-яких $\left\{\psi_{\delta}\right\}_{\delta>0} \subset C^{\infty}\left(0, T ; C_{0}^{\infty}(\Omega)\right)$ таких, що

$$
\psi_{\delta} \rightarrow p \quad \text { сильно в } \quad L^{2}\left(0, T ; H_{0}^{1}(\Omega)\right) .
$$

Оскільки $y \in D$ i

$$
\begin{gathered}
\left|\int_{0}^{T} \int_{\Omega}\left(\nabla \psi_{\delta}, A(x) \nabla \varphi_{\varepsilon}\right)_{\mathbb{R}^{N}} d x d t\right| \\
\leq\left\|\varphi_{\varepsilon}\right\|_{C^{\infty}\left(0, T ; C_{0}^{\infty}(\Omega)\right)}\|A(x)\|_{L^{2}\left(\Omega, \mathbb{S}^{N}\right)}\left\|\psi_{\delta}\right\|_{L^{2}\left(0, T ; H_{0}^{1}(\Omega)\right)},
\end{gathered}
$$

то

$$
\begin{gathered}
\lim _{\delta \rightarrow 0}\left[y+\theta \varphi_{\varepsilon}, \psi_{\delta}\right]=[y, p]+\theta \int_{0}^{T} \int_{\Omega}\left(\nabla p, A(x) \nabla \varphi_{\varepsilon}\right)_{\mathbb{R}^{N}} d x d t \\
=[y, p]-\theta \int_{0}^{T} \int_{\Omega}\left(\nabla \varphi_{\varepsilon}, A(x) \nabla p\right)_{\mathbb{R}^{N}} d x d t .
\end{gathered}
$$


3 вищенаведеного випливає, що

$$
\begin{aligned}
D_{y}^{+} \widehat{L}\left(u, y, \lambda, p, \varphi_{\varepsilon}\right)=2 \lambda \int_{0}^{T} \int_{\Omega}\left(y-y_{d}\right) \varphi_{\varepsilon} d x d t+\int_{0}^{T} \int_{\Omega}\left(\varphi_{\varepsilon}\right)_{t}^{\prime} p d x d t \\
+\int_{0}^{T} \int_{\Omega}\left(\nabla p, \nabla \varphi_{\varepsilon}\right)_{\mathbb{R}^{N}} d x d t-\int_{0}^{T} \int_{\Omega}\left(\nabla \varphi_{\varepsilon}, A(x) \nabla p\right)_{\mathbb{R}^{N}} d x d t
\end{aligned}
$$

для будь-яких $\varphi_{\varepsilon} \in C^{\infty}\left(0, T ; C_{0}^{\infty}(\Omega)\right)$ таких, що

$$
\begin{gathered}
\varphi_{\varepsilon} \rightarrow h \quad \text { сильно в } \quad L^{2}\left(0, T ; H_{0}^{1}(\Omega)\right), \\
\left(\varphi_{\varepsilon}\right)_{t}^{\prime} \rightarrow(h)_{t}^{\prime} \quad \text { сильно в } \quad L^{2}\left(0, T ; H^{-1}(\Omega)\right) .
\end{gathered}
$$

Оскільки $p \in D$, то

$$
\left|\left[p, \varphi_{\varepsilon}\right]\right|:=\left|\int_{0}^{T} \int_{\Omega}\left(\nabla \varphi_{\varepsilon}, A(x) \nabla p\right)_{\mathbb{R}^{N}} d x d t\right| \leq c(p)\left\|\varphi_{\varepsilon}\right\|_{L^{2}\left(0, T ; H_{0}^{1}(\Omega)\right)} .
$$

Отже, права похідна за напрямом $D_{y}^{+} \widehat{L}\left(u, y, \lambda, p, \varphi_{\varepsilon}\right)$ має неперервне продовження для $\varphi \in L^{2}\left(0, T ; H_{0}^{1}(\Omega)\right)$. Крім того, якщо перейти в (3.7) до границі коли $\varepsilon \rightarrow 0$, то отримаємо (3.3).

Наслідок 3.1. Подання (3.3) для узагальненої правої похідної за напрямом $D_{y}^{+} \widehat{L}(u, y, \lambda, p, h)$ мае місце для елементів $y \in L^{2}\left(0, T ; H_{0}^{1}(\Omega)\right)$ таких, що $y \notin D$.

Доведення. Дійсно, у цьому випадку маємо

$$
\begin{gathered}
\widehat{L}(u, y, \lambda, p)=\lambda I(u, y)+\int_{0}^{T} \int_{\Omega} y_{t} p d x d t+\int_{0}^{T} \int_{\Omega}(\nabla p, \nabla y)_{\mathbb{R}^{N}} d x d t \\
-\lim _{\delta \rightarrow 0} \int_{0}^{T} \int_{\Omega}\left(\nabla \psi_{\delta}, A(x) \nabla p\right)_{\mathbb{R}^{N}} d x d t-\langle f, p\rangle_{L^{2}\left(0, T ; H^{-1}(\Omega)\right), L^{2}\left(0, T ; H_{0}^{1}(\Omega)\right)} \\
-\int_{0}^{T} \int_{\Gamma_{2}} u p d \mathcal{H}^{N-1} d t=\lambda I(u, y)+\int_{0}^{T} \int_{\Omega} y_{t} p d x d t \\
+\int_{0}^{T} \int_{\Omega}(\nabla p, \nabla y)_{\mathbb{R}^{N}} d x d t-[p, y]-\langle f, p\rangle_{L^{2}\left(0, T ; H^{-1}(\Omega)\right), L^{2}\left(0, T ; H_{0}^{1}(\Omega)\right)} \\
-\int_{0}^{T} \int_{\Gamma_{2}} u p d \mathcal{H}^{N-1} d t
\end{gathered}
$$

для будь-якої послідовності $\left\{\psi_{\delta}\right\}_{\delta>0} \subset C^{\infty}\left(0, T ; C_{0}^{\infty}(\Omega)\right)$ такої, що

$$
\begin{gathered}
\psi_{\delta} \rightarrow y \quad \text { сильно в } \quad L^{2}\left(0, T ; H_{0}^{1}(\Omega)\right), \\
\left(\psi_{\delta}\right)_{t}^{\prime} \rightarrow(y)_{t}^{\prime} \quad \text { сильно в } \quad L^{2}\left(0, T ; H^{-1}(\Omega)\right) .
\end{gathered}
$$

Очевидно, що (3.4)-(3.6) матимуть такий вигляд:

$$
J=\lim _{\theta \rightarrow+0} \frac{\lim _{\delta \rightarrow 0}\left[\psi_{\delta}+\theta \varphi_{\varepsilon}, p\right]+[p, y]}{\theta},
$$




$$
\begin{gathered}
{\left[\psi_{\delta}+\theta \varphi_{\varepsilon}, p\right]=\int_{0}^{T} \int_{\Omega}\left(\nabla p, A(x) \nabla\left(\psi_{\delta}+\theta \varphi_{\varepsilon}\right)\right)_{\mathbb{R}^{N}} d x d t} \\
=-\int_{0}^{T} \int_{\Omega}\left(\nabla\left(\psi_{\delta}+\theta \varphi_{\varepsilon}\right), A(x) \nabla p\right)_{\mathbb{R}^{N}} d x d t \\
{[p, y]=\lim _{\delta \rightarrow 0} \int_{0}^{T} \int_{\Omega}\left(\nabla \psi_{\delta}, A(x) \nabla p\right)_{\mathbb{R}^{N}} d x d t}
\end{gathered}
$$

для будь-яких $\left\{\psi_{\delta}\right\}_{\delta>0} \subset C^{\infty}\left(0, T ; C_{0}^{\infty}(\Omega)\right)$ таких, що $\psi_{\delta} \rightarrow p$ сильно в просторі $L^{2}\left(0, T ; H_{0}^{1}(\Omega)\right)$. Оскільки $p \in D$, то отримаємо

$$
\lim _{\delta \rightarrow 0}\left[\psi_{\delta}+\theta \varphi_{\varepsilon}, p\right]=-[p, y]-\theta \int_{0}^{T} \int_{\Omega}\left(\nabla \varphi_{\varepsilon}, A(x) \nabla p\right)_{\mathbb{R}^{N}} d x d t
$$

Далі повторюємо доведення, як і в лемі 3.1 .

Наслідок 3.2. Нехай задано четвірку $(u, y, \lambda, p) \in L^{2}\left(0, T ; L^{2}\left(\Gamma_{2}\right)\right) \times D \times \mathbb{R}_{+}$ $\times L^{2}\left(0, T ; H_{0}^{1}(\Omega)\right)$. Припустимо, що $\nabla p \in L^{2}\left(0, T ; L^{\infty}(\Omega)\right)$, а отже, $p \in D$. Тоді узагальнена права похідна за напрямом $D_{y}^{+} \widehat{L}(u, y, \lambda, p, h)$ існуе для кожного напряму $h \in L^{2}\left(0, T ; H_{0}^{1}(\Omega)\right)$ i мае такий вигляд:

$$
\begin{aligned}
D_{y}^{+} \widehat{L}(u, y, \lambda, p, h) & =2 \lambda \int_{0}^{T} \int_{\Omega}\left(y-y_{d}\right) h d x d t+\int_{0}^{T} \int_{\Omega} h_{t}^{\prime} p d x d t \\
& +\int_{0}^{T} \int_{\Omega}(\nabla p, \nabla h)_{\mathbb{R}^{N}} d x d t-\int_{0}^{T} \int_{\Omega}(\nabla h, A(x) \nabla p)_{\mathbb{R}^{N}} d x d t .
\end{aligned}
$$

Доведення. Оскільки $\nabla p \in L^{2}\left(0, T ; L^{\infty}(\Omega)\right)$, то (3.8) можна подати у вигляді

$$
\begin{gathered}
\left|\left[p, \varphi_{\varepsilon}\right]\right|:=\left|\int_{0}^{T} \int_{\Omega}\left(\nabla \varphi_{\varepsilon}, A(x) \nabla p\right)_{\mathbb{R}^{N}} d x d t\right| \\
\leq\|\nabla p\|_{L^{2}\left(0, T ; L^{\infty}(\Omega)\right)}\|A\|_{L^{2}\left(\Omega ; \mathbb{S}^{N}\right)}\left\|\varphi_{\varepsilon}\right\|_{L^{2}\left(0, T ; H_{0}^{1}(\Omega)\right)} .
\end{gathered}
$$

Звідси $p \in D$. Крім того, з леми 3.1 випливає існуванння узагальненої правої похідної за напрямом $D_{y}^{+} \widehat{L}(u, y, \lambda, p, h)$. Слід відзначити, що для заданого $p$ має місце $A \nabla p \in L^{2}\left(0, T ; L^{2}(\Omega)\right)$, а отже, перейшовши до границі в (3.7), отримаємо

$$
[p, h]=\lim _{\varepsilon \rightarrow 0} \int_{0}^{T} \int_{\Omega}\left(\nabla \varphi_{\varepsilon}, A(x) \nabla p\right)_{\mathbb{R}^{N}} d x d t=\int_{0}^{T} \int_{\Omega}(\nabla h, A(x) \nabla p)_{\mathbb{R}^{N}} d x d t .
$$

Наслідок 3.3. Якщо в наслідкові 3.2 замість умови $\nabla p \in L^{2}\left(0, T ; L^{\infty}(\Omega)\right)$ розглянути умови

$$
A(x) \in L^{2+\frac{4}{\gamma}}\left(\Omega ; \mathbb{S}^{N}\right), \nabla p \in L^{2}\left(0, T ; L^{2+\gamma}(\Omega)\right)
$$


для деякого $\gamma \in(0 ; \infty]$, тоді легко переконаємося, що виконуються припущення наслідка 3.2.

Доведення. 3 попереднього наслідка випливає, що достатньо показати $p \in D$. Дійсно, оскільки

$$
\begin{gathered}
|[p, h]|:=\left|\int_{0}^{T} \int_{\Omega}\left(\nabla \varphi_{\varepsilon}, A(x) \nabla p\right)_{\mathbb{R}^{N}} d x d t\right| \\
\leq\left\|\varphi_{\varepsilon}\right\|_{L^{2}\left(0, T ; H_{0}^{1}(\Omega)\right)}\left(\int_{0}^{T} \int_{\Omega}\|\nabla p\|_{\mathbb{R}^{N}}^{2}\|A\|_{\mathbb{S}^{N}}^{2} d x d t\right)^{1 / 2},
\end{gathered}
$$

то, застосувавши нерівність Гельдера для $r=(\gamma+2) / 2$ i $q=1+2 / q$, отримаємо

$$
\begin{gathered}
\int_{0}^{T} \int_{\Omega}\|\nabla p\|_{\mathbb{R}^{N}}^{2}\|A\|_{\mathbb{S}^{N}}^{2} d x d t \leq \int_{0}^{T}\left(\int_{\Omega}\|A\|_{\mathbb{S}^{N}}^{2+\frac{4}{\gamma}} d x\right)^{\frac{\gamma}{2+\gamma}} \\
\left(\int_{\Omega}\|\nabla p\|_{\mathbb{R}^{N}}^{2+\gamma} d x\right)^{\frac{2}{2+\gamma}} d t \leq \int_{0}^{T}\|A\|_{L^{2+\frac{4}{\gamma}\left(\Omega ; \mathbb{S}^{N}\right)}}^{2}\|\nabla p\|_{L^{2+\gamma}(\Omega)}^{2} d t \\
=\|A\|_{L^{2+\frac{4}{\gamma}\left(\Omega ; \mathbb{S}^{N}\right)}}^{2}\|\nabla p\|_{L^{2}\left(0, T ; L^{2+\gamma}(\Omega)\right)}^{2}<+\infty .
\end{gathered}
$$

Отже, $p \in D$. Таким чином, наслідок доведено.

3 вищенаведеного випливає такий результат:

Лема 3.2. Нехай $u \in L^{2}\left(0, T ; L^{2}\left(\Gamma_{2}\right)\right), y \in L^{2}\left(0, T ; H_{0}^{1}(\Omega)\right), \lambda \in \mathbb{R}_{+}, p \in$ $L^{2}\left(0, T ; H_{0}^{1}(\Omega)\right)$ - задані розподілення. Якщо

$$
\nabla p \in L^{2}\left(0, T ; L^{\infty}(\Omega)\right)
$$

$$
\text { або } A(x) \in L^{2+\frac{4}{\gamma}}\left(\Omega ; \mathbb{S}^{N}\right), \nabla p \in L^{2}\left(0, T ; L^{2+\gamma}(\Omega)\right)
$$

для деякого $\gamma \in(0 ; \infty]$, тоді відображення простору $L^{2}\left(0, T ; H_{0}^{1}(\Omega)\right) v \longmapsto$ $\widehat{L}(u, y, \lambda, p, h) \in \mathbb{R} \epsilon$ диферениійовний за Гато $і$ диференціал Гато має вигляд

$$
\left\langle D_{y} \widehat{L}(u, y, \lambda, p), h\right\rangle_{L^{2}\left(0, T ; H^{-1}(\Omega)\right), L^{2}\left(0, T ; H_{0}^{1}(\Omega)\right)}=D_{y}^{+} \widehat{L}(u, y, \lambda, p, h)
$$

для будъ-якого $h \in L^{2}\left(0, T ; H_{0}^{1}(\Omega)\right)$.

Доведення. Нехай задано $(u, y, \lambda, p) \in L^{2}\left(0, T ; L^{2}\left(\Gamma_{2}\right)\right) \times L^{2}\left(0, T ; H_{0}^{1}(\Omega)\right) \times \mathbb{R}_{+}$ $\times L^{2}\left(0, T ; H_{0}^{1}(\Omega)\right)$. 3 наслідків 3.2-3.3 виходить, що $p \in D$, а з леми 3.1 - що величина $[y+\theta h, p]$ означена для всіх $h \in C^{\infty}\left(0, T ; C_{0}^{\infty}(\Omega)\right)$. У свою чергу, 3 (3.14) випливає (3.12), а отже,

$$
D_{y}^{+} \widehat{L}(u, y, \lambda, p, h)=-D_{y}^{+} \widehat{L}(u, y, \lambda, p,-h)
$$

для всіх $h \in C^{\infty}\left(0, T ; C_{0}^{\infty}(\Omega)\right)$. Таким чином, відображення диференційовне за Гато. 
Тепер наведемо необхідний у подальшому результат.

Лема 3.3. Нехай $u \in L^{2}\left(0, T ; L^{2}\left(\Gamma_{2}\right)\right), y \in L^{2}\left(0, T ; H_{0}^{1}(\Omega)\right), \lambda \in \mathbb{R}_{+}, p \in$ $L^{2}\left(0, T ; H_{0}^{1}(\Omega)\right)$ - задані розподілення. Нехай виконуеться умова (3.14). Тоді існуе додатна величина $\varepsilon \in[0,1]$ така, що

$$
\begin{aligned}
\widehat{L}(u, v, \lambda, p)-\widehat{L}(u, y, \lambda, p) & \\
= & \left\langle D_{y} \widehat{L}(u, y+\varepsilon(v-y), \lambda, p), v-y\right\rangle_{L^{2}\left(0, T ; H^{-1}(\Omega)\right), L^{2}\left(0, T ; H_{0}^{1}(\Omega)\right)} \\
= & 2 \lambda \int_{0}^{T} \int_{\Omega}\left(y+\varepsilon(v-y)-y_{d}\right)(v-y) d x d t+\int_{0}^{T} \int_{\Omega}(v-y)_{t}^{\prime} p d x d t \\
+ & \int_{0}^{T} \int_{\Omega}(\nabla p, \nabla(v-y))_{\mathbb{R}^{N}} d x d t-\int_{0}^{T} \int_{\Omega}(\nabla(v-y), A(x) \nabla p)_{\mathbb{R}^{N}} d x d t .
\end{aligned}
$$

\section{4. Необхідні умови оптимальності}

Наразі піде мова про необхідні умови оптимальності для задачі оптимального керування (2.1)-(2.4), але спочатку введемо необхідне в подальшому поняття квазіспряженого оператора.

Означення 4.1. [3] Нехай $u_{\theta} \in L^{2}\left(0, T ; L^{2}\left(\Gamma_{2}\right)\right)$ - допустиме керування і нехай $\varepsilon_{\theta}-$ задана величина, а $y_{\theta}-$ слабкий розв'язок задачі $(2.1)-(2.3)$. Нехай $\left(u_{0}, y_{0}\right) \in \Xi-$ оптимальна пара задачі (2.1)-(2.4). Розподілення $\psi_{\theta}$ будемо називати квазіспряженим станом до $y_{0} \in L^{2}\left(0, T ; H_{0}^{1}(\Omega)\right)$ для фіксованих $\theta \in$ $[0,1], \varepsilon_{\theta} \in[0,1]$, якщо $\psi_{\theta}$ задовольняє тотожність

$$
\begin{aligned}
\int_{0}^{T} \int_{\Omega}\left(\nabla \varphi, A \nabla \psi_{\theta}\right)_{\mathbb{R}^{N}} d x d t-\int_{0}^{T} \int_{\Omega}\left(\psi_{\theta}\right)_{t}^{\prime} \varphi d x d t & \\
& =-2 \lambda \int_{0}^{T} \int_{\Omega}\left(y_{0}-y_{d}\right) \varphi d x d t
\end{aligned}
$$

де $u_{\theta}=u_{0}+\theta\left(\widehat{u}-u_{0}\right), y_{\theta}=y_{0}-\varepsilon_{\theta}\left(y\left(u_{\theta}\right)-y_{0}\right)$. Тут $\widehat{u} \in L^{2}\left(0, T ; L^{2}\left(\Gamma_{2}\right)\right)-$ довільне допустиме керування.

Відзначимо, що для вищеозначеного оператора має місце такий результат:

Твердження 4.1. Нехай $\left(u_{0}, y_{0}\right) \in \Xi-$ оптимальна пара задачі (2.1)-(2.4), a $\left(u_{\theta}, y\left(u_{\theta}\right)\right)$ - допустима пара цієї ж задачі. Тоді для заданого $\theta \in[0,1]$ i $\varepsilon_{\theta} \in[0,1], \psi_{\varepsilon}-$ квазіспряжений оператор до $y_{0} \in L^{2}\left(0, T ; H_{0}^{1}(\Omega)\right)$, якщо $\psi_{\theta} \in D$ і є слабким розв'язком початково-крайової задачі:

$$
\left\{\begin{array}{l}
-\left(\psi_{\theta}\right)_{y}^{\prime}-\operatorname{div}(I-A) \nabla \psi_{\theta}+2 \lambda\left(y_{0}-y_{d}\right)=0 \\
\psi_{\theta}=0 \text { на } \Gamma_{1} \times[0, T] \\
\frac{\partial \psi_{\theta}}{\partial \nu_{I-A}}=0 \text { на } \Gamma_{2} \times[0, T] \\
\psi_{\theta}(T, 0)=0 \text { на } \Omega .
\end{array}\right.
$$


Перед тим як перейти до необхідних умов оптимальності, припустимо, що виконуються такі гіпотези:

(I) Нехай $\left(u_{0}, y_{0}\right) \in \Xi-$ оптимальна пара задачі (2.1)-(2.4); $\widehat{u}-$ довільне допустиме керуванняі $u_{\theta}=u_{0}+\theta\left(\widehat{u}-u_{0}\right)$ для кожного $\theta \in[0,1]$. Тоді існує послідовність відповідних розв'язків крайової задачі (2.1)-(2.3) $y_{\theta}:=$ $y\left(u_{\theta}\right)=\left\{y\left(u_{0}+\theta\left(\widehat{u}-u_{0}\right)\right)\right\}_{\theta \rightarrow 0}$, яка є така, що

$$
y_{\theta} \rightarrow y\left(u_{0}\right) \quad \text { слабко в } L^{2}\left(0, T ; H_{0}^{1}(\Omega)\right),
$$

коли $\theta \rightarrow 0$ і $y \in D$ для достатньо малих $\theta$.

(II) Нехай $\left(u_{0}, y_{0}\right) \in \Xi-$ оптимальна пара задачі (2.1)-(2.4), $\widehat{u}-$ довільне допустиме керуванняі $u_{\theta}=u_{0}+\theta\left(\widehat{u}-u_{0}\right)$ для будь-якого $\theta \in[0,1]$. Тоді для кожного $\theta \in[0,1], \varepsilon_{\theta} \in[0,1]$ існує $\gamma \in(0, \infty]$ :

$$
\begin{gathered}
\nabla \psi_{\theta} \in L^{2}\left(0, T ; L^{\infty}(\Omega)\right) \\
\text { або } A(x) \in L^{2+\frac{4}{\gamma}}\left(\Omega ; \mathbb{S}^{N}\right), \nabla \psi_{\theta} \in L^{2}\left(0, T ; L^{2+\gamma}(\Omega)\right)
\end{gathered}
$$

і послідовність квазіспряжених операторів $\left\{\psi_{\theta}\right\}_{\theta \rightarrow 0} \in$ відносно компактна в сильній топології простору $L^{2}\left(0, T ; H_{0}^{1}(\Omega)\right)$.

Теорема 4.1. Нехай $f \in L^{2}\left(0, T ; H^{-1}(\Omega)\right), y_{d} \in L^{2}\left(0, T ; H_{0}^{1}(\Omega)\right)-$ задані розподілення $i\left(u_{0}, y_{0}\right)$ - оптимальна пара задачі (2.1)-(2.4). Тоді виконання гіпотез (I)-(II) означае існування елементів $\lambda \in \mathbb{R}_{+} i \bar{\psi} \in L^{2}\left(0, T ; H_{0}^{1}(\Omega)\right)$ таких, що $\lambda i \bar{\psi}$ одночасно не дорівнюють $0 i$

$$
\begin{gathered}
\int_{0}^{T} \int_{\Omega}\left(y_{0}\right)_{t}^{\prime} \varphi d x d t+\int_{0}^{T} \int_{\Omega}\left(\nabla \varphi, \nabla y_{0}+A \nabla y_{0}\right)_{\mathbb{R}^{N}} d x d t= \\
=\int_{0}^{T} \int_{\Omega} f \varphi d x d t+\int_{0}^{T} \int_{\Gamma_{2}} u_{0} \varphi d \mathcal{H}^{N-1} d t, \\
\int_{0} \int_{\Omega}(\nabla \varphi, \nabla \bar{\psi}+A \nabla \bar{\psi})_{\mathbb{R}^{N}} d x d t-\int_{0}^{T} \int_{\Omega}(\bar{\psi})_{t}^{\prime} \varphi d x d t= \\
=-2 \lambda \int_{0}^{T} \int_{\Omega}\left(y_{0}-y_{d}\right) \varphi d x d t, \quad \forall \varphi \in C^{\infty}\left(0, T ; C_{0}^{\infty}(\Omega)\right) \\
\int_{0}^{T} \int_{\Gamma_{2}}\left(\widehat{u}-u_{0}\right) u_{0} d \mathcal{H}^{N-1} d t \geq \int_{0}^{T} \int_{\Gamma_{2}}\left(\widehat{u}-u_{0}\right) \bar{\psi} d \mathcal{H}^{N-1} d t .
\end{gathered}
$$


Доведення. Нехай $(\widehat{u}, \widehat{y}) \in \Xi-$ допустима пара вихідної задачі, тоді покладемO

$$
u_{\theta}=u_{0}+\theta\left(\widehat{u}-u_{0}\right)
$$

де $\theta \in[0,1]$, очевидно, що $u_{\theta} \in L^{2}\left(0, T ; L^{2}\left(\Gamma_{2}\right)\right)$ і $u_{\theta} \rightarrow u_{0}$. Нехай $y_{\theta}:=y\left(u_{\theta}\right)=$ $y\left(u_{0}+\theta\left(\widehat{u}-u_{0}\right)\right)-$ розв'язок задачі $(2.1)-(2.3)$. Тоді (II) означає, що

$$
\left(u_{\theta}, y_{\theta}\right) \stackrel{\tau}{\longrightarrow}\left(u_{0}, y_{0}\right),
$$

коли $\theta \rightarrow 0$. Далі розглянемо рівність

$$
\begin{aligned}
\triangle \widehat{L}=\widehat{L}\left(u_{\theta}, y_{\theta}, \lambda, p\right)-\widehat{L}\left(u_{0}, y_{0}, \lambda, p\right)=\widehat{L}\left(u_{\theta}, y_{\theta}, \lambda, p\right) & \\
-\widehat{L}\left(u_{\theta}, y_{0}, \lambda, p\right) & +\widehat{L}\left(u_{\theta}, y_{0}, \lambda, p\right)-\widehat{L}\left(u_{0}, y_{0}, \lambda, p\right) \\
& =\triangle_{y} \widehat{L}\left(u_{\theta}, y_{\theta}, \lambda, p\right)+\triangle_{A} \widehat{L}\left(u_{0}, y_{0}, \lambda, p\right) \geq 0
\end{aligned}
$$

для будь-яких $\theta \in[0,1] \mathrm{i}(\lambda, p) \in \mathbb{R}_{+} \times L^{2}\left(0, T ; H_{0}^{1}(\Omega)\right)$.

Врахувавши подання (3.1)-(3.2), отримаємо

$$
\begin{aligned}
\triangle_{A} \widehat{L}\left(u_{0}, y_{0}, \lambda, p\right)=\widehat{L}\left(u_{\theta}, y_{0}, \lambda, p\right)-\widehat{L}\left(u_{0}, y_{0}, \lambda, p\right) & \\
=\lim _{\delta \rightarrow 0}[ & \left.\widehat{L}\left(u_{\theta}, y_{0}, \lambda, \varphi_{\delta}\right)-\widehat{L}\left(u_{0}, y_{0}, \lambda, \varphi_{\delta}\right)\right]=\lambda \theta^{2}\left\|\widehat{u}-u_{0}\right\|_{L^{2}\left(0, T ; L^{2}\left(\Gamma_{2}\right)\right)}^{2} \\
& +2 \lambda \theta \int_{0}^{T} \int_{\Gamma_{2}}\left(\widehat{u}-u_{0}\right) u_{0} d \mathcal{H}^{N-1} d t-\theta \int_{\Gamma_{2}}^{T}\left(\widehat{u}-u_{0}\right) p d \mathcal{H}^{N-1} d t
\end{aligned}
$$

для будь-якої послідовності $\left\{\varphi_{\delta}\right\}_{\delta>0} \subset C^{\infty}\left(0, T ; C_{0}^{\infty}(\Omega)\right)$ такої, що $\varphi_{\delta} \rightarrow p$ в просторі $L^{2}\left(0, T ; H_{0}^{1}(\Omega)\right)$.

Тепер розглянемо $\triangle_{y} \widehat{L}\left(u_{0}, y_{0}, \lambda, p\right)$ і припустимо, що $p$ належить до простору $L^{2}\left(0, T ; H_{0}^{1}(\Omega)\right)$ і задовольняе (3.14). Тоді за лемою 3.2 існує додатна величина $\varepsilon_{\theta} \in[0,1]$ така, що

$$
\begin{aligned}
& \triangle_{y} \widehat{L}\left(u_{0}, y_{0}, \lambda, p\right)=\widehat{L}\left(u_{\theta}, y_{\theta}, \lambda, p\right)-\widehat{L}\left(u_{\theta}, y_{0}, \lambda, p\right) \\
& =\left\langle D_{y} \widehat{L}\left(u_{\theta}, y_{0}+\varepsilon_{\theta}\left(y_{\theta}-y_{0}\right), \lambda, p\right), y_{\theta}-y_{0}\right\rangle_{L^{2}\left(0, T ; H^{-1}(\Omega)\right), L^{2}\left(0, T ; H_{0}^{1}(\Omega)\right)} \\
& =2 \lambda \int_{0}^{T} \int_{\Omega}\left(y_{0}+\varepsilon_{\theta}\left(y_{\theta}-y_{0}\right)-y_{d}\right)\left(y_{\theta}-y_{0}\right) d x d t+\int_{0} \int_{\Omega}\left(y_{\theta}-y_{0}\right)_{t}^{\prime} p d x d t \\
& \quad+\int_{\Omega}\left(\nabla p, \nabla\left(y_{\theta}-y_{0}\right)\right)_{\mathbb{R}^{N}} d x d t-\int_{\Omega}\left(\nabla\left(y_{\theta}-y_{0}\right), A \nabla p\right)_{\mathbb{R}^{N}} d x d t .
\end{aligned}
$$

Таким чином, враховуючи (4.9) і (4.10), беручи до уваги властивість (3.14) і 
лему 3.3 , перепишемо рівність (4.8) таким чином:

$$
\begin{gathered}
\triangle \widehat{L}=2 \lambda \int_{0}^{T} \int_{\Omega}\left(y_{0}+\varepsilon_{\theta}\left(y_{\theta}-y_{0}\right)-y_{d}\right)\left(y_{\theta}-y_{0}\right) d x d t+\int_{0}^{T} \int_{\Omega}\left(y_{\theta}-y_{0}\right)_{t}^{\prime} p d x d t \\
+\int_{\Omega}\left(\nabla p, \nabla\left(y_{\theta}-y_{0}\right)\right)_{\mathbb{R}^{N}} d x d t-\int_{\Omega}\left(\nabla\left(y_{\theta}-y_{0}\right), A \nabla p\right)_{\mathbb{R}^{N}} d x d t \\
+\lambda \theta^{2}\left\|\widehat{u}-u_{0}\right\|_{L^{2}\left(0, T ; L^{2}\left(\Gamma_{2}\right)\right)}^{2}+2 \lambda \theta \int_{0}^{T} \int_{\Gamma_{2}}\left(\widehat{u}-u_{0}\right) u_{0} d \mathcal{H}^{N-1} d t \\
-\theta \int_{0}^{T} \int_{\Gamma_{2}}\left(\widehat{u}-u_{0}\right) p d \mathcal{H}^{N-1} d t
\end{gathered}
$$

Враховуючи (4.3), означимо елемент $p$ в (4.11) як квазіспряжений оператор до стану $y_{0} \in L^{2}\left(0, T ; H_{0}^{1}(\Omega)\right)$, тобто покладемо $p=\psi_{\theta}$, де $\psi_{\theta}$ задовольняє таку рівність:

$$
\begin{gathered}
\int_{0}^{T} \int_{\Omega} \varphi_{t}^{\prime} \psi_{\theta} d x d t+\int_{\Omega}(\nabla \varphi, \nabla p-A \nabla p)_{\mathbb{R}^{N}} d x d t \\
=\int_{\Omega}(\nabla \varphi, \nabla p-A \nabla p)_{\mathbb{R}^{N}} d x d t-\int_{0}^{T} \int_{\Omega} \varphi\left(\psi_{\theta}\right)_{t}^{\prime} d x d t \\
=2 \lambda \int_{0}^{T} \int_{\Omega}\left(y_{0}+\varepsilon_{\theta}\left(y_{\theta}-y_{0}\right)-y_{d}\right) \varphi d x d t
\end{gathered}
$$

де $\varphi=y_{\theta}-y_{0}$. Таким чином,

$$
\begin{aligned}
\frac{\triangle \widehat{L}}{\theta}= & \frac{\widehat{L}\left(u_{\theta}, y_{\theta}, \lambda, \psi_{\theta}\right)-\widehat{L}\left(u_{0}, y_{0}, \lambda, \psi_{\theta}\right)}{\theta}=\lambda \theta\left\|\widehat{u}-u_{0}\right\|_{L^{2}\left(0, T ; L^{2}\left(\Gamma_{2}\right)\right)}^{2} \\
& +2 \lambda \int_{0}^{T} \int_{\Gamma_{2}}\left(\widehat{u}-u_{0}\right) u_{0} d \mathcal{H}^{N-1} d t-\int_{0}^{T} \int_{\Gamma_{2}}\left(\widehat{u}-u_{0}\right) \psi_{\theta} d \mathcal{H}^{N-1} d t \geq 0,
\end{aligned}
$$

тут $\widehat{u} \in L^{2}\left(0, T ; L^{2}\left(\Gamma_{2}\right)\right)$.

Залишилось перейти до границі в (4.12) і (4.13), для цього відзначимо, що

$$
\begin{gathered}
u_{\theta} \rightarrow u_{0}, \quad \text { коли } \theta \rightarrow 0, \\
y_{\theta} \rightarrow y_{0} \quad \text { в } \quad L^{2}\left(0, T ; H_{0}^{1}(\Omega)\right), \\
\text { iснуе } \bar{\psi} \in L^{2}\left(0, T ; H_{0}^{1}(\Omega)\right): \quad \psi_{\theta} \rightarrow \bar{\psi} \quad \text { в } \quad L^{2}\left(0, T ; H_{0}^{1}(\Omega)\right)
\end{gathered}
$$


Тоді

$$
\lim _{\theta \rightarrow 0} \frac{\triangle \widehat{L}}{\theta}=2 \lambda \int_{0}^{T} \int_{\Gamma_{2}}\left(\widehat{u}-u_{0}\right) u_{0} d \mathcal{H}^{N-1} d t-\int_{0}^{T} \int_{\Gamma_{2}}\left(\widehat{u}-u_{0}\right) \bar{\psi} d \mathcal{H}^{N-1} d t \geq 0 .
$$

Звідси випливає, що

$$
2 \lambda \int_{0}^{T} \int_{\Gamma_{2}}\left(\widehat{u}-u_{0}\right) u_{0} d \mathcal{H}^{N-1} d t \geq \int_{0}^{T} \int_{\Gamma_{2}}\left(\widehat{u}-u_{0}\right) \bar{\psi} d \mathcal{H}^{N-1} d t
$$

Отже, якщо в (4.12) перейти до границі за $\theta \rightarrow 0$, то отримаємо (4.5), що і потрібно було встановити.

\section{Бібліографічні посилання}

1. Иоффе А. Д. Теория экстремальных задач / А. Д. Иоффе, В. М. ТихомировМ.: Наука, 1974. - 479 c.

2. Горбонос С. О. Варіаційні розв'язки задачі оптимального керування з необмеженими коефіцієнтами / Горбонос С.О., Когут П.I. // Вісник Дніпропетр. ун-ту. Серія: Моделювання. - 2013. - Вип. 5. - С. 69-83.

3. Horsin T. Optimal $L^{2}$-Control Problem in Coefficients for a Linear Elliptic Equation / T. Horsin, P. Kogut, submitted to Mathematical Control and Related Fields, 2013, 1-60. (arXiv:1306.2513)

4. Salsa S. Partial Differential Equations in Action: From Modelling to Theory /

S. Salsa - Milan: Springer-Verlag, 2008. - 556 p. 